\title{
Simulation and Optimization of Discharging Process of Powder Material Suction Vehicle Based on Fluent
}

\author{
Wu yong-hai, Bao Hai-tao, Fan Qin-man \\ Faculty of Traffic Engineering, Huaiyin Institute of Technology, Huai'an, China \\ wuchinese2011@163.com
}

\begin{abstract}
Keywords: Powder material suction vehicle; Two- phase flow; Simulation; Fluent
Abstract. For gas-solid two-phase flow material suction and discharging process, the computational fluid dynamics numerical method was used to carry out numerical simulation through RNG turbulence model and Euler two-phase flow model. The material discharging system of a certain Powder material suction vehicle was taken as the research object, the fluidized tank flow model was established using Gambit software. Fluent software was used to calculate flow field distribution and volume distribution of solid phase in fluidized tanks when the discharge port is at a different distance from the air distribution plate. The results show that the discharge port has obvious influence on unloading speed. The best discharging effect is achieved when the discharge port is $100 \mathrm{~mm}$ away from the air distribution plate, and the discharging process is the most stable.
\end{abstract}

\section{Introduction}

Powder material suction vehicle is a professional logistics vehicle engaged in bulk material transportation. It is used for closed transportation of powdered and granular dry bulk materials such as mineral powder, fly ash, grain, etc.. It is of great significance to reduce the loss of bulk materials, reduce loading and unloading costs, save packaging materials, improve working conditions of workers, improve working efficiency and protect the environment. At present, the design of the Powder material suction vehicle car is mainly dependent on the experience design method, and it is difficult to calculate the performance of the suction and discharge. With the development of computer technology, the use of Computational Fluid Dynamics (CFD) to study the flow characteristics in the tank and the mixing effect of the powder has been gradually appreciated. For gas-solid two-phase flow material suction and discharging process, the computational fluid dynamics numerical method was used to carry out numerical simulation through RNG turbulence model and Euler two-phase flow model. The material discharging system of a certain Powder material suction vehicle was taken as the research object, the fluidized tank flow model was established using Gambit software. Fluent software was used to calculate flow field distribution and volume distribution of solid phase in fluidized tanks when the discharge port is at a different distance from the air distribution plate. The results show that the discharge port has obvious influence on unloading speed. The best discharging effect is achieved when the discharge port is $100 \mathrm{~mm}$ away from the air distribution plate, and the discharging process is the most stable.

\section{Mathematical model of flow field of suction and drainage system}

Gas - solid two-phase flow is composed of particles and air. Using the double Euler method, both phases are considered as a continuum. That is, the particles are considered as a pseudo-fluid to form a two-fluid model. This model introduces the volume fraction of two phases into the traditional mass, momentum, and energy conservation equations to form a closed set of control equations. The equations are as follows:

\section{Momentum equation}

$$
\frac{\partial}{\partial_{t}}\left(\varepsilon_{k} \rho_{k}\right)+\nabla \cdot\left(\varepsilon_{k} \rho_{k}\right)=0
$$


where: $\varepsilon$ is turbulent energy consumption efficiency, $\mathrm{m}^{2} \cdot \mathrm{s}^{-3}$; subscript $\mathrm{k}$ represents a certain phase; $\mathrm{vk}$ is $\mathrm{k}$ phase velocity, $\mathrm{m} / \mathrm{s} ; \rho$ is density, $\mathrm{kg} \cdot \mathrm{m}^{-3} ; \mathrm{t}$ is time, $\mathrm{s} ; \nabla$ is a Hamiltonian operator; the gradient of the number field is $\operatorname{grad} \mathrm{A}=\nabla \mathrm{A}$; the divergence of the vector field is $\operatorname{div} \mathrm{A}=\nabla \cdot \mathrm{A}$.

Gas phase:

$$
\frac{\partial}{\partial_{t}}\left(\varepsilon_{g} \rho_{g} v_{s}\right)+\nabla \cdot\left(\varepsilon_{g} \rho_{g} v_{g} v_{g}\right)=-\varepsilon_{g} \nabla p+\varepsilon_{g} \rho_{g} g+\nabla \tau_{g}-\beta\left(v_{g}-v_{s}\right)
$$

Particle phase:

$$
\frac{\partial}{\partial_{t}}\left(\varepsilon_{s} \rho_{s} v_{s}\right)+\nabla \cdot\left(\varepsilon_{s} \rho_{s} v_{s} v_{s}\right)=-\varepsilon_{s} \nabla p+\varepsilon_{s} \rho_{s} g-\nabla+p_{s} \nabla \cdot \tau_{s}-\beta\left(v_{g}-v_{s}\right)
$$

where: subscript $g$ represents the gas phase, $s$ represents the particle phase; $\mathrm{v}_{\mathrm{g}}, \mathrm{v}_{\mathrm{s}}$ are the velocity vector of the gas phase and the solid phase, $\mathrm{m} / \mathrm{s} ; \beta$ is the coefficient of phase resistance; $\mathrm{p}$ is the pressure, $\mathrm{Pa}$;

$$
\begin{aligned}
& \tau_{g}=\varepsilon_{g} \xi_{g} \nabla \cdot v_{g} I+2 \varepsilon_{g} \mu_{g} S_{g} \\
& S_{g}=\frac{1}{2}\left[\nabla v_{g}+\left(\nabla v_{g}\right)^{T}\right]-\frac{1}{3} \nabla v_{g} I \\
& \tau_{s}=\varepsilon_{s} \xi_{s} \nabla v_{s} I+2 \varepsilon_{s} \mu_{s} \\
& S_{s}=\frac{1}{2}\left[\nabla v_{s}+\left(\nabla v_{s}\right)^{T}\right]-\frac{1}{3} \nabla v_{s} I \\
& \beta=\frac{3}{4} C_{d} \frac{\varepsilon_{g} \varepsilon_{s} \rho\left|v_{g}-v_{s}\right|}{d_{s}} \varepsilon_{s}^{-2.65}, \quad\left(\varepsilon_{g} \geq 0.8\right) \\
& C_{d}=\frac{24}{R e}\left[1+0.15 R e^{0.687}\right],(R e<1000) \\
& C_{d}=0.44,(R e \geq 1000) \\
& \beta=\frac{150 \varepsilon_{s}\left(1-\varepsilon_{g}\right) \mu_{g}}{\varepsilon_{g} d_{s}^{2}}+\frac{1.75 \rho_{g} \varepsilon_{s}\left|v_{g}-v_{s}\right|}{d_{s}}, \quad\left(\varepsilon_{g}<0.8\right)
\end{aligned}
$$

where: $\mathrm{I}$ is the unit tensor; $\mu$ is the viscosity coefficient, $\mathrm{Pa} \cdot \mathrm{s}^{-1} ; \tau$ is the viscous stress tensor, $\mathrm{N} \cdot \mathrm{m}^{-2} ; \beta$ is the coefficient of phase resistance; $\mathrm{S}$ is the velocity deformation tensor, $\mathrm{s}^{-1} ; \xi$ is the volume viscosity, $\mathrm{Pa} \cdot \mathrm{s}$; $\mathrm{Cd}$ is the drag coefficient; Re is the Reynolds number.

\section{Particle kinetic equation}

Radial distribution function:

$$
g_{0}=\left[1-\left(\varepsilon_{s} / \varepsilon_{s, \max }\right)^{1 / 3}\right]^{-1}
$$

where: $\mathrm{g}_{0}$ is a radial distribution function.

Particle pressure:

$$
p_{s}=\varepsilon_{s} \rho_{s}\left[1+2(1+e) \varepsilon_{s} g_{0}\right]
$$

where: $\mathrm{e}$ is the elastic recovery factor.

Particle phase volume viscosity: 


$$
\xi_{s}=\frac{4}{3} \varepsilon_{s}^{2} \rho_{s} d_{s} g_{0}(1+e)\left(\frac{\Theta_{s}}{\pi}\right)^{1 / 2}
$$

where: $\Theta_{s}$ is the particle temperature (the kinetic energy of particle turbulence), $\mathrm{m}^{2} \cdot \mathrm{s}^{-2} ; \mathrm{d}_{\mathrm{s}}$ is the particle diameter, $m$.

Particle phase viscosity:

$$
\mu_{s}=\frac{4}{5} \varepsilon_{s}^{2} \rho_{s} d_{s} g_{0}(1+e)\left(\frac{\Theta_{s}}{\pi}\right)^{1 / 2}+\frac{10 \rho_{s} d_{s} \sqrt{\Theta_{s} \pi}}{-6 \varepsilon_{s}(1+e) g_{0}} \cdot\left[1+\frac{4}{5} g_{0} \varepsilon_{s}(1+e)\right]^{2}
$$

Particle temperature transfer equation:

$$
\frac{3}{2}\left[\frac{\partial}{\partial_{t}}\left(\rho_{s} \varepsilon_{s} \Theta_{s}\right)+\nabla \cdot\left(\rho_{s} \varepsilon_{s} v_{s} \Theta_{s}\right)\right]=\left(-p_{s} I+\tau_{s}\right) \cdot \nabla v_{s}-\nabla \cdot\left(K_{\Theta_{s}} \nabla \Theta_{s}\right)-\gamma_{\Theta s}+\phi_{g s}
$$

where: $\mathrm{K}$ is the turbulent kinetic energy, $\mathrm{J} \cdot \mathrm{kg}^{-1} ; \gamma$ is the collision energy dissipation rate, $\mathrm{kg} \cdot \mathrm{m}^{-1} \cdot \mathrm{s}^{-3} ; \phi_{g s}$ is the energy transfer between the two phases, $\mathrm{kg} \cdot \mathrm{m}^{-1} \cdot \mathrm{s}^{-3}$.

\section{The simulation of the suction and exhaust vehicle discharging process and the result analysis}

Took the body of straight powder material suction tank as an example, it chose two-dimensional characteristic cross section as a model of the process of the discharge of the fluid-bed. The process of discharge was only considering the single value factor, only the effect of the distance between the discharge port and the air distribution plate on the unloading process during the material tanker unloading is simulated.

Set three difference distance between the air distribution plate and the discharge nozzle as 50mm, $100 \mathrm{~mm}$ and $150 \mathrm{~mm}$. Used the 'Fluent' to simulate the process. The result was showed as the picture 1 to picture 3 .

From the material volume flow figure, when the distance was $100 \mathrm{~mm}$, as the picture 2 showed, the flow of the material volume was more stable than the two other situations. The volume kept at $33 \sim 40 \mathrm{~kg} / \mathrm{s}$ most of the time and the average volume was $36.2 \mathrm{~kg} / \mathrm{s}$. When the distance was $50 \mathrm{~mm}$, the volume had obvious changes showed as picture 1, this kind of phenomenon showed that the process of the material outflow was very unstable, and a swirl would be produced at the discharge port, the material flowed back the material warehouse after flowing into the discharge port. The phenomenon was called as eddy current which caused by the fierce flowing of the gas material mixture at the discharge port. When the distance was set as $150 \mathrm{~mm}$, the flow of the material volume had certain changes, showed as the picture 3, the discharge port was relatively high that caused the flowing process was not very stable. From the experience of the simulation calculation, the flow of the gas material mixture was not stable at the air distribution plate and its liquid level. At the position of the liquid level, the flow was influenced by the wave of the liquid level directly and the mixture was not very stable. The phenomenon was not very obvious at the early stage of the discharge, but it would cause the flow through the discharge port reducing obviously at the middle and late stage.

In general, the location of the discharge pipe has a significant effect on the discharge speed. The discharge speed will be relatively low if the position of the discharge pipeline is too high, the pressure is small and the volume fraction of the material is small. If the low position of the pipeline mouth leads to a very low volume fraction of material in the area between the discharge pipe mouth and the air distribution plate. The gas will form a "short circuit" between the air distribution plate and the discharge pipe, which does not play a very good role in carrying the material particles. By comparing, when the distance from the discharge port is $100 \mathrm{~mm}$, the discharging effect is the best and the discharging process is the most stable.

From the figure, it can be see that when the discharge port is $100 \mathrm{~mm}$ away from the air distribution plate. The total process time is $160 \mathrm{~s}$ and the flow rate is $36.2 \mathrm{~kg} / \mathrm{s}$. While the discharge time of the other 
two discharge conditions is about 185s. The average flow rate is $27 \mathrm{~kg} / \mathrm{s}$ and $29 \mathrm{~kg} / \mathrm{s}$. It can be seen that the position of the discharge port has a significant influence on the discharge speed.

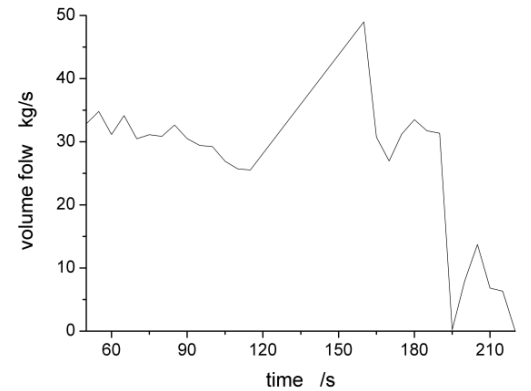

(a)flow figure

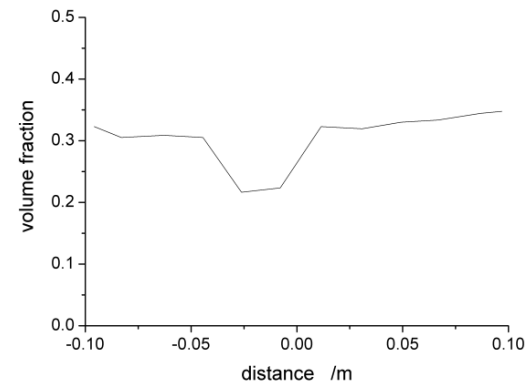

(b)volume fraction(100s)

Fig. 1 Discharge port distance cloth plate $50 \mathrm{~mm}$

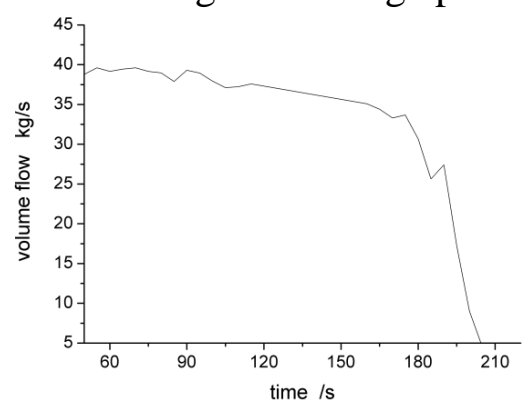

(a)flow figure

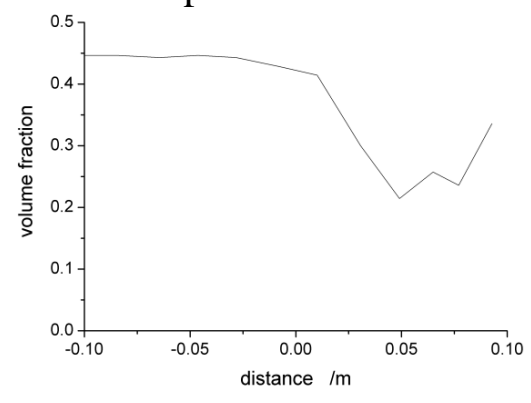

(b)volume fraction(100s)

Fig.2 Discharge port distance cloth plate $100 \mathrm{~mm}$

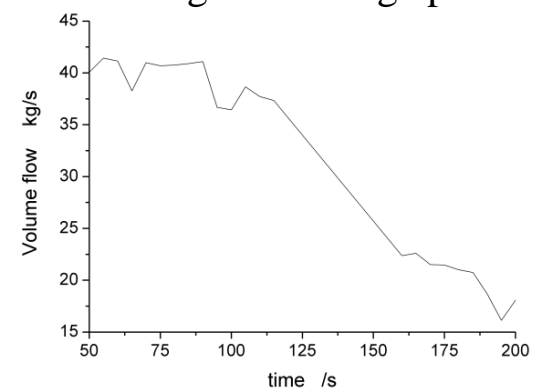

(a)flow figure

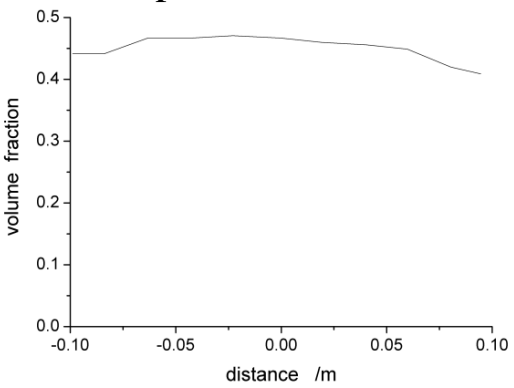

(b)volume fraction(100s)

Fig.3 Discharge port distance cloth plate $150 \mathrm{~mm}$

\section{References}

[1] Xiang Zhao, Chance Glenn, Zhigang Xiao, Sijun Zhang. CFD development for macro particle simulations. International Journal of Computational Fluid Dynamics, Vol.5 (2014), p.142

[2] G.R. Kasat, A.R. Khopkar, V.V. Ranade, A.B. Pandit. CFD simulation of liquid-phase mixing in solid-liquid stirred reactor. Chemical Engineering Science. Vol.15 (2008), p.431

[3] G. Montante, F. Magelli. Modelling of solids distribution in stirred tanks: analysis of simulation strategies and comparison with experimental data. International Journal of Computational Fluid Dynamics. Vol.3 (2005), p.189

[4] G. Micale, G. Montante, F. Grisafi, A. Brucato, J. Godfrey. CFD Simulation of Particle Distribution in Stirred Vessels. Chemical Engineering Research and Design. Vol. 3(2000), p.152 